\title{
Rechtsgeschichte
}

http://www.rg-rechtsgeschichte.de/rg4

$\operatorname{Rg} 42004 \quad 57-60$

Zitiervorschlag: Rechtsgeschichte Rg 4 (2004)

http://dx.doi.org/10.12946/rg04/057-060

\section{Hermann Klenner}

\section{Warum ausgerechnet keine Rechtsgeschichte?}

Mommsens Block! 


\section{Abstract}

Legal history is an indispensable discipline within the study of law! According to Theodor Mommsen, one of the most influential persons in German jurisprudence, it is necessary that history and law converge in order to allow an insight into how law functions. To explore the reasons and functions of older legal orders which paved the way for modern law means to understand the past of law as integrating part of its presence and future. Therefore the knowledge of how law developed is a condition for understanding today's law, implementing law, and further developing of law. Because a jurist is trained to think not only de lege lata but also de lege ferenda, he badly needs a precise memory of the brilliance as well as the deficiencies of former norms and sentences. Without experience no insight! If a lawyer is not aware of former solutions of legal problems and their dubious nature he ceases to be a member of the scientific community. Legal history thereby can also be a help to get rid of the past and to set out for new lands. 


\section{Warum ausgerechnet keine Rechtsgeschichte?}

Mommsens Block!

Die I 852 gehaltene Zürcher Antrittsvorlesung des vor genau einhundert Jahren verstorbenen Theodor Mommsen enthielt den (wenn man ihn nicht gerade savignyisch interpretiert) provokativen Gedanken, dass die Entwicklung eines lebendigen Rechts durch nichts mehr vorangetrieben werde als durch seine historische Erforschung. Auch wenn Stolleisens biographisches Juristenlexikon von 1995 sich nicht die Ehre gibt, für il grande Teodoro ein paar Zeilen zu erübrigen, gehört er doch zu den erfolgreichsten Rechtshistorikern aller Zeiten. Sein Credo: Um "Einsicht in das innere Räderwerk des Rechts « zu nehmen, bedürfe es der »Verschmelzung von Geschichte und Jurisprudenz «. ${ }^{\mathbf{I}}$

Wem freilich alle Epochen gleich nahe zu Gott angesiedelt erscheinen, dem kann Rechtsgeschichte nur zur frommen Unterhaltung dienen, als Ritual eines Omnia ad maiorem Dei gloriam. Dessen säkularisierte GleichgültigkeitsVersion von der gleichen Gültigkeit alles Vergangenen, wonach die großen Rechtsdenker allemal dasselbe dachten, verweist ebenso wie deren nihilistische Lesart, wonach die Probleme der Wissenschaft nicht gelöst, sondern nur von anderen Problemen abgelöst werden, die Rechtsgeschichte in das Luxusland einer Orchideendisziplin - solange man sich diesen schönen Schein noch leisten kann.

Rechtsgeschichte mag zwar, emotional gesehen, identitätsbildend wirken, wie die Taten (und Untaten) der weltgeschichtlichen Individuen auch; wissenschaftlich gesehen aber ist dieses looking for identity into the past jedenfalls nicht legitimitätsbegründend, da doch alles, was entstanden ist, auch eines schönen oder unschö- nen Tages zugrunde zu gehen wert und bestimmt ist. Aber auch wer geltendes Recht lediglich aus gewesenem Recht konkludiert und sich daher künftiges Recht nur aus gegenwärtigem Recht induziert vorzustellen vermag, hat für Rechtsgeschichte im eigentlichen Sinne keine Verwendung; er substituiert bloß rechtshistorische Abläufe durch normlogische Kontinuitätsketten. Recht aber nur auf Recht zu beziehen ist selbstreferenziell und daher ungeschichtlich. ${ }^{2}$

Um den Begründungsdruck, dem die Rechtsgeschichte als eigenständige Wissenschaftsdisziplin ausgesetzt ist, noch zu verschärfen, wie den Zweifel an der Existenzberechtigung steuerfinanzierter Rechtshistoriker auch, mag gleich zu Beginn eingeräumt werden, dass es unmöglich ist, ohne ein weises Vergessen von Vergangenem das gegenwärtig Erforderliche zu erwirken (und nicht nur zu erdulden). Aber schon die Frage, $o b$ etwas aus der Überfülle des Überlieferten (und was?) im Interesse des Gegenwärtigen nicht vergessen werden dürfe, erzwingt eine methodenbewusste Vorgehensweise. Diese ermöglicht uns einerseits, übermäßige Komplexität durch Ausmusterungen zu reduzieren und andererseits, das auf uns gekommene Gesetzgebungs- und Rechtsprechungsmaterial nicht wie einen Schutthaufen pauschal $\mathrm{zu}$ entsorgen, sondern wie einen Steinbruch nutzbringend für den Straßen- und den Häuserbau, d.h. für eine zu verbessernde Rechtsordnung zu verwerten.

An Veränderungen, sogar Entwicklungen kommt keiner vorbei, der aus der Wahrheit seiner Sinne lebt. Insofern gilt immer: Everything goes, und somewhere geht es auch. Aber geht es auch vorwärts? Oder liegt das Goldene Zeitalter

I Theodor Mommsen, Gesammelte Schriften, Bd. 3, Berlin 1907 , 600; DERs., Reden und Aufsätze, Berlin 1905,36 .

2 So Gerd Roellecke, Theoretische Überlegungen zum Verhältnis von Rechtsgeschichte und Rechtspraxis, in: Der praktische Nutzen der Rechtsgeschichte, hg. von JÖRN ECKERT, Heidelberg 2003 , 430. 
auf ewig hinter uns, und wir erleben ohne Aussicht auf ein Anderswerden jene Jahre, von denen es bei Hesiod heißt, ${ }^{3}$ da das Volk die Frevel der Herrscher büßt? Gehört »Fortschritt «, wie Engels dachte, ${ }^{4}$ zum Wesen der Menschheit oder, wie Marx meinte, ${ }^{5}$ zumindest zum Prinzip demokratischer Verfassungen? Oder ist er nur die Illusion dessen, der zu schieben glaubt, obwohl er bloß geschoben wird? Ist »Fortschritt « vielleicht lediglich eine Leerformel auf höchstem Abstraktionsniveau, konsensstiftend dem Scheine nach, tatsächlich aber dissensverdeckend? Folgenlos ist jedenfalls die Vergötzung der Zeitfolge an sich, die Ernst Bloch in seinem Berliner Akademie-Vortrag von 1955 als Unlust denunzierte, selber ein Rad im Uhrwerk der Geschichte zu sein. ${ }^{6}$ Hegels Entschluss, einerseits dem Kommando des Weltgeistes zu parieren und andererseits diesem Avanceriesen Schuhpech, das ihn festhalten soll, anschmieren $\mathrm{zu}$ helfen, ${ }^{7}$ setzt zumindest den guten Glauben an die säkulare Variante eines personalen Gottes voraus.

Die (objektiven) Bedingungen der Gesellschaft können so wenig hinwegdekretiert werden, wie die Verwirklichung der (subjektiven) Ziele der Gesellschaftsakteure herbeidekretiert werden kann. Juristische Gesetze vermögen immer nur innerhalb einer gewissen Bandbreite Vehikel oder Bremse historischer Emanzipationsvorgänge zu sein. Zutreffend ist jedoch gegen die bekannte Behauptung der Historischen Rechtsschule, die Rechtsgeschichte würde durch innere still wirkende Kräfte bewegt und die einzelnen Rechtsinstitute würden organisch entfaltet werden, eingewandt worden, dass in der Realität die Rechtsentwicklung in der Diskussion über alternative Vorschläge und in der Entscheidung für bestimmte Lösungen vor sich geht. ${ }^{8}$ Die Erinnerung an Glanz und Elend früherer Gesetzgebungsvorhaben und Gerichtsusancen ist doch keine Zwangsjacke, die abzuwerfen aufs Innigste zu wünschen sei. Erinnerung, klug gehandhabt, kann doch auch eine Vorstellung vom Möglichen, von Widerstand und von Befreiung ergeben, kann uns zu eingreifendem Denken und Handeln verhelfen. Darauf zu beharren, scheint mir in einer Zeit, die nicht durch Fortschrittsoptimismus, sondern durch dessen Antagonisten geprägt ist, und noch dazu in unserer Wissenschaftsdisziplin, die - wie keine andere - unter Konservatismusverdacht steht und dem Progress höchstens nachzuhumpeln pflegt, ${ }^{9}$ besonders angebracht zu sein, auch wenn dem Zeitgeist entgegenzudenken Sisyphostugenden erfordert.

Warum sollten ausgerechnet diejenigen, denen die Entwicklung des Rechts wie niemandem sonst anheimgegeben ist, die Brücken zu seiner Vergangenheit abbrechen? Warum sollten sie sich des Weges zum geltenden Recht schämen, der es doch dahin geführt hatte, wo es nun ist? Konvertiten pflegen ihre eigene Vergangenheit zu »bewältigen ", indem sie die alten Götter verbrennen und deren Jünger kreuzigen. Sie sind die geborenen Autodafé-Veranstalter und Bücherverbrenner. Ein Rechtswissenschaftler hingegen begreift die Vergangenheit des Rechts als Moment seiner Gegenwart, und mit dieser also auch seiner Zukunft. Wenn einem Rechtsdenker die bisherigen normativen Problemlösungen deren Fragwürdigkeit vollständig verdecken, hört er auf, ein Rechtsdenkender zu sein. Warum eigentlich sollten die Juristen Wittgensteins metaphorischer Forderung nachgeben und die Leitern ihres Erkenntnisfortschritts wegwerfen, nachdem diese ihnen den Aufstieg ermöglicht haben ? $^{\text {Io }}$ Sollten sie sogar das Wissen darüber elidieren, wie man einen Aufstieg ermöglichende Leitern baut?

So wie ein Jurist ein guter Jurist nur dann sein kann, wenn er sein De-lege-lata-Denken mit

\footnotetext{
3 Hesiod, Werke und Tage, Düsseldorf, Zürich I997, Verse $260 \mathrm{f}$. 4 Karl Marx, Friedrich Engels, Gesamtausgabe [MEGA], Bd. I/3, Berlin $1985,576$.

5 MEGA I/2, 6I.

6 ERnst Bloch, Differenzierungen im Begriff Fortschritt, Berlin I957, 7.

7 Briefe von und an Hegel, hg. von JohanNes Hoffmeister, Bd. 2, Berlin I970, 86.
}

8 Helmut Coing, Aufgaben des Rechtshistorikers (Sitzungsberichte der wissenschaftlichen Gesellschaft an der Johann Wolfgang von Goethe-Universität Frankfurt am Main I3, Nr. 8), Wiesbaden I 976, I 7 I.

9 So zutreffend Ralf Dreier, Das Fortschrittsproblem in rechtstheoretischer Sicht, in: Rechtswissenschaft und Rechtsentwicklung, hg. von UlRICH IMMENGA (Göttinger rechtswissenschaftliche Studien, Bd. I I I), Göttingen I980, I. io Ludwig Wittgenstein, Tractatus logico-philosophicus, Frankfurt I 984 , II 5 . 
einem De-lege-ferenda-Denken, seinen Wirklichkeitssinn mit einem Möglichkeitssinn zu paaren vermag, so gehört seine Kenntnis des Gewordenseins von Recht und Gesetz zu deren Verständnis-, Verwirklichungs-, aber auch zu deren Entwicklungsbedingungen. Alles auf Anfang zu stellen, empfiehlt sich nicht; denn dann bliebe man vor der Gegenwart stecken. Aber das Risiko, das bei jeder Veränderung des Rechts unvermeidlich ist, vermindert sich, wenn man den Übermut der Neuerungssüchtigen mit dem abkühlenden Hinweis auf gewesene Erfahrungen diszipliniert. Was auch immer die Systemtheoretiker sagen und die Polit-Praktiker mit ihren Überheblichkeitsallüren zu tun bereit sein mögen: Das Recht einer Gesellschaft gehört nicht zu deren Fungibilien. Gewiss können die Wegbereiter von Einst nicht die Wegweiser von Heute sein; aber die Dialektik von Herkunft und $\mathrm{Zu}$ kunft zu leugnen, führte zu einem Verzicht auf die Erfahrungen, die guten und die schlechten, die eine gewesene oder eine gescheiterte Rechtsordnung zumindest als ein Gedanken-Reservoir für diejenigen bietet, die nicht dem legislatorischen Größenwahn verfallen sind, neue Gesetze als creationes ex nibilo produzieren zu können. Und wenn es sich nur darum handeln würde, das Unabgegoltene wenn schon nicht zur Geltung zu bringen, dann wenigstens als Bewusstseinsstachel zu erhalten.

Auf einer intellektuellen Ebene vermögen sich die Gedankengebilde (zu denen Rechtsordnungen doch auch zählen) der verschiedenen Epochen durchaus wie Zeitgenossen zu treffen. Die gegenwärtigen Gesetzgeber können an die Gesetze ihrer Vorgänger Fragen stellen. Auch wenn sie diese Fragen selbst beantworten müssen, hilft ihnen das überlieferte Normativmaterial dennoch, moderne Probleme zu lösen. Impliziert doch jede wissenschaftliche Arbeit die
Kooperation mit den Erfahrungen und den Erkenntnissen nicht nur der Gegenwartsdenker. Wie anders sollte man Rechtsnormen und Gerichtsurteile namhaft machen, hinter die nicht zurückgegangen werden darf? Andererseits: Auch wenn jede Gegenwart (zunächst) eine Noterbin der Vergangenheit ist, muss es doch so nicht bleiben; Rechtsgeschichte kann nämlich sogar als ein Versuch verstanden werden, sich das Vergangene vom Halse zu schaffen.

Um mit wenigstens drei Beispielen konkret zu werden:

Es läuft doch gerade die mit Fallstricken geschmückte Goldhagen-Debatte zu der Frage, ob die Juden oder die Römer schuld gewesen seien an der Hinrichtung des Jesus von Nazareth; »Der Jurist « Mommsen hatte längst gegen die damaligen Auffassungen »der Theologen « Protest eingelegt, da die so genannten Pilatus-Akten von einem Verfasser stammten, der keinerlei Ahnung vom jüdisch-römischen Strafprozess gehabt habe; ${ }^{\text {II }}$ nach damaligem Juden-Recht hätte nämlich Jesus gesteinigt werden müssen und nicht, wie nach Römer-Recht, gekreuzigt werden dürfen. ${ }^{\text {I2 }}$

Wäre es nicht in der unsäglichen Diskussion darüber, ob man im Ermittlungsverfahren als ultima ratio wieder die Folter einsetzen solle, sinnvoll, sich der von Rechtshistorikern längst ermittelten Erfahrungen mit diesem Menschenwie Wahrheitskiller zu vergewissern, statt auf absolute Werte zu rekurrieren; denn diese sind in Bekenntnissen, nicht in Erkenntnissen fundiert, also nicht verhandelbar - Glaube trennt, Vernunft eint.

Und gehört es nicht zur Verantwortung von Juristen, auch rechtshistorisch gegen die kriegsverbrecherischen Unternehmungen der Jetztzeit zu argumentieren, die nach dem Zweiten Weltkrieg etablierte, erstmals universale Völker-

\footnotetext{
I I Theodor Mommsen, Gesammelte Schriften, Bd. 3, Berlin I907, 430 ("Die Pilatus-Akten «, I902); DANIEL Jonah GoldhaGEN, Die katholische Kirche und der Holocaust, Berlin 2002, 345 , 44I.

I 2 gl. Mommsen, Römisches Strafrecht (I 899), Berlin I95 5, 240 f., 9 I 8 .
} 
rechtsordnung unter den Vorbehalt jener berüchtigten clausula rebus sic stantibus $\mathrm{zu}$ stellen? Über Jahrtausende widerspiegelten Bacons Erfahrungssätze die blutige Wirklichkeit: Among the causes of war few are more popular than the putting down of tyrannies [...]. As long as men are men and as long as reason is reason a just fear will be a just cause of a preventive war. ${ }^{\mathrm{I3}}$ Zurück ins Mittelalter? -

Ohne Eigenschaften mag Mann (in der Literatur vielleicht) leben können, gewiss jedoch nicht ohne Gedächtnis. Es ist aber gerade das peculium der Rechtshistoriker, das für Rechtswissenschaft und Rechtspraxis spezifische Gedächtnis verfügbar zu halten. Insofern ist neben der gewiss prävalierenden Rechtsdogmatik die Rechtsgeschichte (wie aus anderen Gründen die Rechtssoziologie, die Rechtsvergleichung, die Rechtstheorie und die Rechtsphilosophie auch) eine unabdingbare Juristendisziplin.

Da ich mit Mommsen begonnen habe, will ich mit ihm enden, wenn auch mit ihm als einem Medium der Literatur. Vom bedeutendsten deutschen Dramatiker nach Brecht ist ein Poem, Mommsens Block überliefert. Vermutlich durch einen Brief Nietzsches über den Brand im Haus Mommsen, Machstraße 8 in Charlottenburg bei Berlin, angeregt, aus dem zu entnehmen war, dass der Historiker mit den vielleicht mächtigsten Vorarbeiten unter den jetzt lebenden Gelehrten immer wieder in die Flammen hineingestürzt sei, um seine Exzerpte zu retten - »als ich die Geschichte hörte, drehte sich mir das Herz im Leibe um, und noch jetzt leide ich physisch, wenn ich daran denke. Aber was geht mich Mommsen an? Ich bin ihm gar nicht gewogen ${ }^{\mathbf{1 4}}$-, beginnt Heiner Müller, über die angebotenen Antworten auf die Frage zu sinnieren, warum es keinen vierten Band von Mommsens Römischer Geschichte gebe? Sei das Manuskript mitverbrannt oder habe ihr Autor, seines heißgeliebten Caesars Tod zu schildern, nicht mehr die Leidenschaft gehabt? Und warum?

Recht oder Unrecht: Es ist die Unerbittlichkeit der Menschheitsgeschichte, die, ausgelöst durch Mommsens Wohnungsbrand, Müller reflektiert. ${ }^{{ }^{5}}$ In einem Dreisprung ohnegleichen parallelisiert er den Untergang des Imperium Romanum mit dem jenes Deutschen Reiches, dessen Geburtshelfer Bismarck zugleich sein Totengräber war, aber auch mit dem »Sturz der asiatischen Despotie Produkt / Einer falschen Lektüre und fälschlich genannt / Sozialismus nach dem großen Historiker des Kapitals«, einem Untergang, dem auch Mommsens Wissenschaftsakademie zum Opfer gefallen sei, während sein Denkmal nun wieder auf dem Sockel »Vor der Universität benannt nach Humboldt / Von den Machthabern einer Illusion« stehe. Als Koda offeriert Müller das Catch-as-catch-can im Deutschland des zu Ende gehenden zwanzigsten Jahrhunderts: Als »Helden der Neuzeit « agieren die »Lemuren des Kapitals Wechsler und Händler «, und es schlagen »die Armen auf die Ärmsten ein«. - Machen wir uns nichts vor: Es ist unsere Wirklichkeit, in der wir warten »for the earthquakes to come".

Hermann Klenner*
I 3 Francis Bacon, The Works, London I 857-I 874 , Bd. I, 532; 4, 329; I 4, 477.

I4 Friedrich Nietzsche, Werke, hg. von Karl Schlechta, Bd. 3 , München I977, I I64 (Brief vom I8. Juli I 880 an Peter Gast). Vgl. H. Klenner, Il grande Teodoro, der Jurist, in: Sitzungsberichte der Leibniz-Sozietät 59 (2003) I I 5.
I 5 Vgl. Heiner Müller, "Mommsens Block « [Dezember I992], in: Die Gedichte, Frankfurt I998, 257-263.

\footnotetext{
* Em. Professor für Rechtsphilosophie und Rechtsgeschichte an der Humboldt-Universität zu Berlin
} 